\title{
野生稻与亚洲栽培稻的遗传多样性
}

\author{
段世华 ${ }^{1,2}$ 李绍清 ${ }^{2}$ 李绍波 ${ }^{3}$ 朱英国 ${ }^{2, *}$
}

${ }^{1}$ 井冈山大学生命科学学院, 江西吉安 $343009 ;{ }^{2}$ 武汉大学植物发育生物学教育部重点实验室, 湖北武汉 $430072 ;{ }^{3}$ 南昌大学生命科学 学院, 江西南昌 330031

摘 要: 为评价野生稻与亚洲栽培稻的遗传多样性及其变异关系, 56 对 SSR 引物被用于研究广泛地理分布的 55 份普 通野生稻(其中 32 份 O. rufipogon 和 23 份 O. nivara)和 25 份亚洲栽培稻(14 份 indica 和 11 份 japonica)样本。298 个 多态性位点被检出, 占总扩增等位点的 $98.68 \%$ 。野生稻多态性位点的百分比(平均达 $91 \%$ )及 Nei's 遗传多样性值( $h$ ) 明显高于亚洲栽培稻，表明普通野生稻比亚洲栽培稻具更丰富的遗传多样性。UPGMA 聚类分析显示野生稻的两个类 群(O. rufipogon 和 O. nivara) 关系密切, 但在遗传上存在明显的分化, 支持其作为两个独立物种的分类观点。许多普 通野生稻中籼粳分化尽管不很明显, 然而亚洲栽培稻的籼粳亚种分化是明显的。亚洲栽培稻与多年生普通野生稻 $(O$. rufipogon)关系更为密切, 符合异源起源的遗传分化模式。

关键词: SSR；亚洲栽培稻；普通野生稻；遗传多样性

\section{Genetic Diversity of Wild Rice and Cultivated Rice}

\author{
DUAN Shi-Hua ${ }^{1,2}$, LI Shao-Qing ${ }^{2}$, LI Shao-Bo ${ }^{3}$, and ZHU Ying-Guo ${ }^{2, *}$ \\ ${ }^{1}$ College of Life Sciences, Jinggangshan University, Ji'an 343009, China; ${ }^{2}$ Key Laboratory of Ministry of Education for Plant Developmental Bio- \\ logy, Wuhan University, Wuhan 430072, China; ${ }^{3}$ College of Life Sciences, Nanchang University, Nanchang 330031, China
}

\begin{abstract}
To evaluate the genetic diversity and variation of common wild rice and Asian cultivated rice, 55 accessions of common wild rice (32 accessions of $O$. rufipogon and 23 accessions of $O$. nivara) and 25 cultivars of Asian cultivated rice (14 indica and 11 japonica) collected worldwide were analyzed by 56 SSR primer pairs. The polymorphic loci percentage was $98.68 \%$ and 298 polymorphic loci were detected. A comparison of the polymorphic loci percentage and Nei's genetic diversity $(h)$ indicated that the genetic diversity of common wild rice was obviously higher than that of Asian cultivated rice. The UPGMA cluster analysis revealed that there was a close relationship between $O$. rufipogon and O. nivara, but distinct genetic differentiation was detected between them, supporting the piont of view that they are two independent species. The results indicated that the indica-japonica differentiation was not very apparent in many wild rice whereas it was obvious in Asian cultivated rice. According to the cluster analysis, there was a closer consanguineous relationship between Asian cultivated rice and O. rufipogon, which supported the diphyletic origin hypotheses.
\end{abstract}

Keywords: SSR; Cultivated rice; Common wild rice; Genetic diversity

水稻是中国乃至全世界最重要的粮食作物之一, 迄今已经有 11000 多年的栽培驯化历史 ${ }^{[1]}$ 。亚洲栽 培稻(Oryza sativa L.)在其栽培驯化过程中由于适应 不同的农业生态环境, 形成了丰富的遗传多样性和 明显的遗传分化，产生了适应于不同农业生态环境 种植的籼型(indica)和粳型(japonica) 栽培稻亚种 ${ }^{[2]}$ 。 稻属的野生物种中含有丰富的优良性状基因, 是水 稻栽培品种改良的重要遗传资源。野生稻(包括一年 生的 Oryza nivara Sharma et. Shastry 和多年生的
Oryza rufipogon Griff.)被认为是亚洲栽培稻的祖先 种, 广泛分布于亚洲热带和亚热带地区。栽培稻与 野生稻(O. nivara 和 O. rufipogon)具有相同的基因组 型(AA 基因组), 遗传关系密切, 近年来许多研究者 分别从形态、同工酶和 DNA 水平对其遗传分化及变 异模式进行了广泛的探讨 ${ }^{[3-7]}$, 然而, 依然存在三方 面争议: (i)野生稻O. nivara 和 O. rufipogon 的分类分 级处理; (ii)亚洲栽培稻的起源, 是来源于一年生类 型野生稻，还是多年生类型野生稻或者是多年生与

本研究由国家重点基础研究发展计划(973 计划)项目(2006CB101700), 江西省教育厅基础研究项目(2007321), 江西省科技厅指导性项目资助。 “通讯作者(Corresponding author): 朱英国, E-mail: zhuyg@public.wh.hb.cn; Tel: 027-68756530 
一年生类型的中间型野生稻; (iii)栽培稻的籼粳亚种 是单次起源还是多次起源。

微卫星 DNA(microsatellite DNA)或称简单序列 重复(simple sequence repeat, SSR)在水稻染色体上 具有广泛的分布 ${ }^{[8-9]}$ 。由于其具有检测等位基因数目 多, 扩增结果稳定, 重复性好, 检测手段简便易行 等优点, 并且 SSR 标记为共显性遗传, 能很好地揭 示供试个体或群体内完整的遗传信息, 因此被广泛 应用于亲缘关系密切或一些快速辐射类群间遗传变 异关系的分析 ${ }^{[10-11]}$ 。本研究在基因组 DNA 水平上, 对野生稻(包括 O. rufipogon 和 O. nivara)与亚洲栽 培稻的遗传多样性及其变异关系进行了分析, 同时 也对亚洲栽培稻的起源与分化进行了探讨。

\section{1 材料与方法}

\section{1 植物材料}

采用了来自不同稻作国家和地区的亚洲栽培稻 样品 25 份, 其中 O. sativa ssp. indica 14 份, O. sativa ssp. japonica 11 份, 以及来自广泛地理分布的野生 稻样本 55 份(包括 32 份 O. rufipogon 和 23 份 $O$. nivara)。野生稻样本均由菲律宾国际水稻研究所遗 传资源中心(Genetic Resources Center, International Rice Research Institute, Los Banos, Philippines)提供 (表 1), 所有栽培稻种子样品均为本实验室收集保存 (表 2)。

\section{2 方法}

\subsubsection{DNA 的提取 基因组总 DNA 均从各供试}

表 1 野生稻样品号、来源及类型

Table 1 Accession number, origins, and types of wild rice used in this study

\begin{tabular}{|c|c|c|c|c|c|c|c|}
\hline $\begin{array}{l}\text { 编号 } \\
\text { No. }\end{array}$ & $\begin{array}{c}\text { 品系号 } \\
\text { Accession No. }\end{array}$ & $\begin{array}{c}\text { 来源 } \\
\text { Origin } \\
\end{array}$ & $\begin{array}{l}\text { 类型 }^{\mathrm{a}} \\
\text { Type }^{\mathrm{a}} \\
\end{array}$ & $\begin{array}{l}\text { 编号 } \\
\text { No. }\end{array}$ & $\begin{array}{c}\text { 品系号 } \\
\text { Accession No. }\end{array}$ & $\begin{array}{c}\text { 来源 } \\
\text { Origin } \\
\end{array}$ & $\begin{array}{l}\text { 类型 }{ }^{\mathrm{a}} \\
\text { Type }^{\mathrm{a}}\end{array}$ \\
\hline 1 & 103835 & Bangladesh & $\mathrm{N}$ & 29 & 104057 & China & $\mathrm{R}$ \\
\hline 2 & 103841 & Bangladesh & $\mathrm{N}$ & 30 & 105400 & China & $\mathrm{R}$ \\
\hline 3 & 105712 & Cambodia & $\mathrm{N}$ & 31 & 101193 & Taiwan, China & $\mathrm{R}$ \\
\hline 4 & 105728 & Cambodia & $\mathrm{N}$ & 32 & 101974 & India & $\mathrm{R}$ \\
\hline 5 & 106309 & Cambodia & $\mathrm{N}$ & 33 & 104709 & India & $\mathrm{R}$ \\
\hline 6 & 103821 & China & $\mathrm{N}$ & 34 & 105349 & India & $\mathrm{R}$ \\
\hline 7 & 103824 & China & $\mathrm{N}$ & 35 & 105079 & India & $\mathrm{R}$ \\
\hline 8 & 81857 & India & $\mathrm{N}$ & 36 & 106083 & India & $\mathrm{R}$ \\
\hline 9 & 101978 & India & $\mathrm{N}$ & 37 & 106158 & Laos & $\mathrm{R}$ \\
\hline 10 & 102163 & India & $\mathrm{N}$ & 38 & 106145 & Laos & $\mathrm{R}$ \\
\hline 11 & 101971 & India & $\mathrm{N}$ & 39 & 106161 & Laos & $\mathrm{R}$ \\
\hline 12 & 104705 & India & $\mathrm{N}$ & 40 & 105491 & Malaysia & $\mathrm{R}$ \\
\hline 13 & 106148 & Laos & $\mathrm{N}$ & 41 & 106036 & Malaysia & $\mathrm{R}$ \\
\hline 14 & 106153 & Laos & $\mathrm{N}$ & 42 & 106386 & Myanmar & $\mathrm{R}$ \\
\hline 15 & 106344 & Myanmar & $\mathrm{N}$ & 43 & 105696 & Nepal & $\mathrm{R}$ \\
\hline 16 & 106345 & Myanmar & $\mathrm{N}$ & 44 & 105698 & Nepal & $\mathrm{R}$ \\
\hline 17 & 105704 & Nepal & $\mathrm{N}$ & 45 & 106264 & Papua New Guinea & $\mathrm{R}$ \\
\hline 18 & 105706 & Nepal & $\mathrm{N}$ & 46 & 106281 & Papua New Guinea & $\mathrm{R}$ \\
\hline 19 & 103415 & Sri Lanka & $\mathrm{N}$ & 47 & 101075 & Philippines & $\mathrm{R}$ \\
\hline 20 & 103419 & Sri Lanka & $\mathrm{N}$ & 48 & 103305 & Philippines & $\mathrm{R}$ \\
\hline 21 & 104612 & Sri Lanka & $\mathrm{N}$ & 49 & 105568 & Philippines & $\mathrm{R}$ \\
\hline 22 & 104650 & Thailand & $\mathrm{N}$ & 50 & 103423 & Sri Lanka & $\mathrm{R}$ \\
\hline 23 & 104659 & Thailand & $\mathrm{N}$ & 51 & 104599 & Sri Lanka & $\mathrm{R}$ \\
\hline 24 & 105887 & Bangladesh & $\mathrm{R}$ & 52 & 100219 & Thailand & $\mathrm{R}$ \\
\hline 25 & 103844 & Bangladesh & $\mathrm{R}$ & 53 & 101941 & Thailand & $\mathrm{R}$ \\
\hline 26 & 105902 & Bangladesh & $\mathrm{R}$ & 54 & 106166 & Vietnam & $\mathrm{R}$ \\
\hline 27 & 105735 & Cambodia & $\mathrm{R}$ & 55 & 106516 & Vietnam & $\mathrm{R}$ \\
\hline 28 & 106321 & Cambodia & $\mathrm{R}$ & & & & \\
\hline
\end{tabular}

${ }^{\mathrm{a}} \mathrm{R}$ : O. rufipogon; N: O. nivara 
表 2 栽培稻品种名、来源及类型

Table 2 The name, origins, and types of rice cultivars used in this study

\begin{tabular}{|c|c|c|c|c|c|c|c|}
\hline $\begin{array}{l}\text { 编号 } \\
\text { No. }\end{array}$ & $\begin{array}{c}\text { 品种名 } \\
\text { Name of cultivar }\end{array}$ & $\begin{array}{c}\text { 来源 } \\
\text { Origin }\end{array}$ & $\begin{array}{l}\text { 类型 }^{\mathrm{a}} \\
\text { Type }^{\mathrm{a}}\end{array}$ & $\begin{array}{l}\text { 编号 } \\
\text { No. }\end{array}$ & $\begin{array}{c}\text { 品种名 } \\
\text { Name of cultivar }\end{array}$ & $\begin{array}{c}\text { 来源 } \\
\text { Origin }\end{array}$ & $\begin{array}{l}\text { 类型 }^{\mathrm{a}} \\
\text { Type }^{\mathrm{a}}\end{array}$ \\
\hline 56 & 南特号 Nantehao & $\begin{array}{l}\text { Guangdong, } \\
\text { China }\end{array}$ & I & 69 & Peta & Indonesia & I \\
\hline 57 & 矮脚南特 Aijiaonante & $\begin{array}{l}\text { Guangdong, } \\
\text { China }\end{array}$ & I & 70 & R1409 & Jiangsu, China & $\mathrm{J}$ \\
\hline 58 & 胜泰 1 号 Shengtai 1 & $\begin{array}{l}\text { Guangdong, } \\
\text { China }\end{array}$ & I & 71 & L-湘晴 L-xiangqing & Shanghai, China & $\mathrm{J}$ \\
\hline 59 & 湘早籼 Xiangzaoxian 21 & Hunan, China & I & 72 & $\mathrm{C} 418$ & Liaoning, China & $\mathrm{J}$ \\
\hline 60 & 广陆矮 4 号 Guanglu’ai 4 & $\begin{array}{l}\text { Guangdong, } \\
\text { China }\end{array}$ & I & 73 & 中花 11 Zhonghua 11 & Beijing, China & $\mathrm{J}$ \\
\hline 61 & 珍汕 97 Zhenshan 97 & Jiangxi, China & I & 74 & PC311 & Hunan, China & $\mathrm{J}$ \\
\hline 62 & 特青 Teqing & $\begin{array}{l}\text { Guangdong, } \\
\text { China }\end{array}$ & I & 75 & R1229 & Tianjin, China & $\mathrm{J}$ \\
\hline 63 & $93-11$ & Jiangsu, China & I & 76 & 日本晴 Nipponbare & Japan & $\mathrm{J}$ \\
\hline 64 & 明恢 63 Minghui 63 & Fujian, China & I & 77 & 秋光 Qiuguang & Japan & $\mathrm{J}$ \\
\hline 65 & $\begin{array}{l}\text { 台中本地 } 1 \text { 号 } \\
\text { Taichung native } 1\end{array}$ & Taiwan, China & I & 78 & 台北 309 Taibei 309 & Taiwan, China & $\mathrm{J}$ \\
\hline 66 & 密阳 23 Milyang 23 & Korea & I & 79 & Balila & Italy & $\mathrm{J}$ \\
\hline 67 & IR36 & IRRI & I & 80 & Lemont & USA & $\mathrm{J}$ \\
\hline 68 & IR64 & IRRI & I & & & & \\
\hline
\end{tabular}

${ }^{\mathrm{a}} \mathrm{I}$ : indica; $\mathrm{J}$ : japonica

水稻材料的新鲜叶片中提取, 方法为修改的 CTAB 法 $^{[12]}$ 。

1.2.2 SSR 扩增反应及扩增产物的电泳检测共 选用了 56 对大致均匀分布于水稻基因组 12 条染色 体上的 SSR 引物进行 PCR 扩增(表 3)。2 $25 \mu \mathrm{L}$ 反应 体系中含 $10 \mathrm{mmol} \mathrm{L}^{-1}$ Tris- $\mathrm{HCl}, 50 \mathrm{mmol} \mathrm{L}^{-1} \mathrm{KCl}$, $0.001 \%$ Gelatin, $2.5 \mathrm{mmol} \mathrm{L}^{-1} \mathrm{MgCl}_{2}, 200 \mu \mathrm{mol} \mathrm{L}^{-1}$ dNTPs (TaKaRa), 0. $3 \mu \mathrm{mol} \mathrm{L}^{-1}$ SSR 引物, $1 \mathrm{U} \mathrm{Taq}$ DNA polymerase (TaKaRa), $25 \mathrm{ng}$ 模板 DNA。9 $94^{\circ} \mathrm{C}$ 预 变性 $5 \mathrm{~min}, 40$ 个循环, 每循环 $94^{\circ} \mathrm{C} 1 \mathrm{~min}$, $55 \sim 62^{\circ} \mathrm{C}$ (各引物所用的退火温度根据推荐设置) 1 $\min , 72^{\circ} \mathrm{C} 1.5 \mathrm{~min}$, 最后 $72^{\circ} \mathrm{C}$ 延伸 $10 \mathrm{~min}$ 。PCR 扩增 在 PTC-100 Programmable Thermal Controller PCR 仪 上完成。将扩增产物在含有 $0.1 \mathrm{~g} \mathrm{~L}^{-1}$ 溴化乙锭的 $4.0 \%$ 琼脂糖凝胶上电泳分离。DL2000 为 DNA 分子 量标准。将电泳产物在凝胶成像系统(Gel DOC 2000, BIO-RAD Laboratories-Segrate, Milan, Italy)的紫外 灯下观察、照相并保存图片。

1.2.3 数据统计与分析 每对 SSR 引物检测 1 个 位点, 视每条多态性带为 1 个等位基因; 将观测到 的每条带视为 1 个性状, 有此带时赋值为“ 1 ”, 无此 带时赋值为“ “”，按 Nei 的方法求算每两份材料间的 遗传距离 $D$, 即 $D=1-\left[2 M_{x y} /\left(M_{x}+M_{y}\right)\right]$, 式中 $M_{x}$ 和 $M_{y}$ 分别为 $X$ 和 $Y$ 材料的总片段数, $M_{x y}$ 为两材料 的公共片段数。对各物种(或亚种)类群内的 SSR 扩
增位点数、多态性位点的百分数、Shannon 指数 $(I)$ 以及 Nei's 无偏倚遗传多样性值 $(h)[h=(N / N-1)(1-$ $\left.\left.\sum P_{i}^{2}\right)\right]^{[13]}$ ，通过人工或 1.31 版本的 POPGENE 软件进 行统计 ${ }^{[14]}$ 。通过所有品系的两两配对组合形成的数 据矩阵, 并利用 NTSYS-PC $2.02^{[15]}$ 软件计算相似系 数。根据相似系数的数据集, 利用非加权配对算术 平均法(unweighted pair group method with arithmetic average, UPGMA)构建遗传关系树状图。根据 Bostein 等 ${ }^{[16]}$ 描述和 Anderson 等 ${ }^{[17]}$ 修改的对于自花传粉物种 的多态性信息量(polymorphism information content, PIC)值来描述等位基因的多态性, PIC $=1-\sum p_{i j}{ }^{2}, p_{i j}$ 是指标记 $i$ 的第 $j$ 种模式的频度直至第 $n$ 种模式的总 和。

\section{2 结果与分析}

\subsection{SSR 引物的 PCR 扩增}

56 对 SSR 引物分别对 80 份亚洲栽培稻和野生 稻材料进行了 PCR 扩增, 大部分引物产生了丰富的 遗传多态性变异模式。图 1 是 RM286 对 80 份水稻 材料 PCR 扩增产生的凝胶电泳图谱。56 个 SSR 引 物对在 80 份水稻材料中扩增所产生的等位基因数 有相当大的变化, 其变化范围为 $2 \sim 8$ 个等位点, 平均 每个引物对产生的等位基因为 5.4 个。每个 SSR 引物 对所揭示的多态性信息量 PIC 表现出非常大的变化, 在 $0.431 \sim 0.864$ 之间(表 3)。56 个 SSR 引物对在所 
表 3 检测的 SSR 位点及其在供试水稻材料中的等位基因数和多态性信息量

Table 3 SSR locus, allele number, and polymorphism information content (PIC) in rice materials of this study

\begin{tabular}{|c|c|c|c|c|c|c|c|}
\hline $\begin{array}{l}\text { 位点 } \\
\text { Locus }\end{array}$ & $\begin{array}{c}\text { 染色体 } \\
\text { Chromosome }\end{array}$ & $\begin{array}{l}\text { 等位基因数 } \\
\text { No. of alleles }\end{array}$ & $P I C^{\mathrm{a}}$ & $\begin{array}{l}\text { 位点 } \\
\text { Locus }\end{array}$ & $\begin{array}{c}\text { 染色体 } \\
\text { Chromosome }\end{array}$ & $\begin{array}{l}\text { 等位基因数 } \\
\text { No. of alleles }\end{array}$ & $P I C^{\mathrm{a}}$ \\
\hline RM5 & 1 & 6 & 0.597 & RM214 & 7 & 6 & 0.710 \\
\hline RM212 & 1 & 5 & 0.532 & RM11 & 7 & 7 & 0.828 \\
\hline RM220 & 1 & 5 & 0.618 & RM10 & 7 & 5 & 0.722 \\
\hline RM243 & 1 & 7 & 0.613 & RM248 & 7 & 6 & 0.817 \\
\hline RM323 & 1 & 2 & 0.471 & RM230 & 8 & 5 & 0.686 \\
\hline RM154 & 2 & 5 & 0.802 & RM407 & 8 & 4 & 0.523 \\
\hline RM263 & 2 & 5 & 0.713 & RM544 & 8 & 2 & 0.431 \\
\hline RM250 & 2 & 4 & 0.604 & RM556 & 8 & 7 & 0.732 \\
\hline RM208 & 2 & 5 & 0.675 & RM419 & 8 & 6 & 0.649 \\
\hline RM282 & 3 & 3 & 0.622 & RM105 & 9 & 5 & 0.760 \\
\hline RM85 & 3 & 4 & 0.591 & RM285 & 9 & 5 & 0.652 \\
\hline RM156 & 3 & 2 & 0.503 & RM189 & 9 & 6 & 0.721 \\
\hline RM168 & 3 & 7 & 0.781 & RM271 & 10 & 4 & 0.720 \\
\hline RM307 & 4 & 7 & 0.831 & RM228 & 10 & 7 & 0.828 \\
\hline RM255 & 4 & 5 & 0.639 & RM330A & 10 & 5 & 0.732 \\
\hline RM303 & 4 & 6 & 0.762 & RM333 & 10 & 8 & 0.823 \\
\hline RM401 & 4 & 5 & 0.627 & RM258 & 10 & 6 & 0.757 \\
\hline RM216 & 4 & 4 & 0.722 & RM20 & 11 & 7 & 0.817 \\
\hline RM559 & 4 & 5 & 0.632 & RM287 & 11 & 7 & 0.832 \\
\hline RM161 & 5 & 6 & 0.641 & RM144 & 11 & 6 & 0.764 \\
\hline RM507 & 5 & 7 & 0.821 & RM552 & 11 & 7 & 0.697 \\
\hline RM440 & 5 & 5 & 0.546 & RM332 & 11 & 5 & 0.650 \\
\hline RM574 & 5 & 6 & 0.723 & RM286 & 11 & 6 & 0.721 \\
\hline RM225 & 6 & 5 & 0.769 & RM254 & 11 & 7 & 0.805 \\
\hline RM3 & 6 & 5 & 0.722 & RM415 & 12 & 5 & 0.631 \\
\hline RM235 & 6 & 6 & 0.805 & RM260 & 12 & 6 & 0.864 \\
\hline RM435 & 6 & 8 & 0.823 & RM463 & 12 & 4 & 0.596 \\
\hline RM557 & 6 & 5 & 0.621 & RM277 & 12 & 3 & 0.560 \\
\hline
\end{tabular}

${ }^{\text {a }}$ PIC: polymorphism information content.

有的水稻材料中共检测出 302 个等位点, 其中 298 个 为多态性等位点(占 $98.68 \%$ )。在 56 对 SSR 引物的 扩增图谱中有大约 5.3\%的杂合位点在供试水稻材料 中被检测出, 这可能是亚洲栽培稻与普通野生稻在 地理分布上的一定重叠及品种之间相互异交或者基 因渗入所致。另外也有一些引物在某些样品中产生 零扩增位点, 如引物 RM307 在野生稻样本“103423” 和引物 RM559 在栽培粳稻“日本晴”中经过多次反复 扩增均没有扩增产物(图片未显示)。

2.2 亚洲栽培稻与野生稻种群内遗传多样性分 析

根据 56 个 SSR 引物对在 80 个水稻材料中扩增 出的 302 个等位点构成的数据矩阵, 对亚洲栽培稻 (indica 和 japonica) 和野生稻(O. nivara 和 O. rufipogon) 各类群内的遗传多样性参数进行了统计分
析。在所有供试物种或亚种类群中扩增出的多态性 位点百分比(percentage of polymorphic bands, PPB) 为 $98.68 \%$, 其变化范围为 $59.79 \%$ 93.28\%, 野生稻 的两个类群的多态性位点明显高于栽培稻的籼粳类 群。在不同物种或者亚种类群中观察到的等位基因 数 $(\mathrm{Na})$ 呈现出明显的变化, 拥有最高值的是 $O$. rufipogon (1.7273), 最低值的是 O. sativa ssp. japonica (1.3953)。所有水稻材料的总 Nei's 遗传多样 性值 $(h)$ 为 0.1905 , 表现出相当程度的变化, 具有最 高值的是 O. rufipogon (0.1937), 而具有最低值的是 O. sativa ssp. indica (0.1061)。有效等位基因数 $(\mathrm{Ne})$ 和 Shannon's 信息指数 $(I)$ 在不同物种或者亚种类群 中的变化趋势与 Nei's 遗传多样性值 $(h)$ 的变化趋势 相类似, 所有供试水稻材料总的有效等位基因数 $(\mathrm{Ne})$ 和 Shannon's 信息指数 $(I)$ 分别是 1.2968 和 0.3048 (表 4$)$ 。 

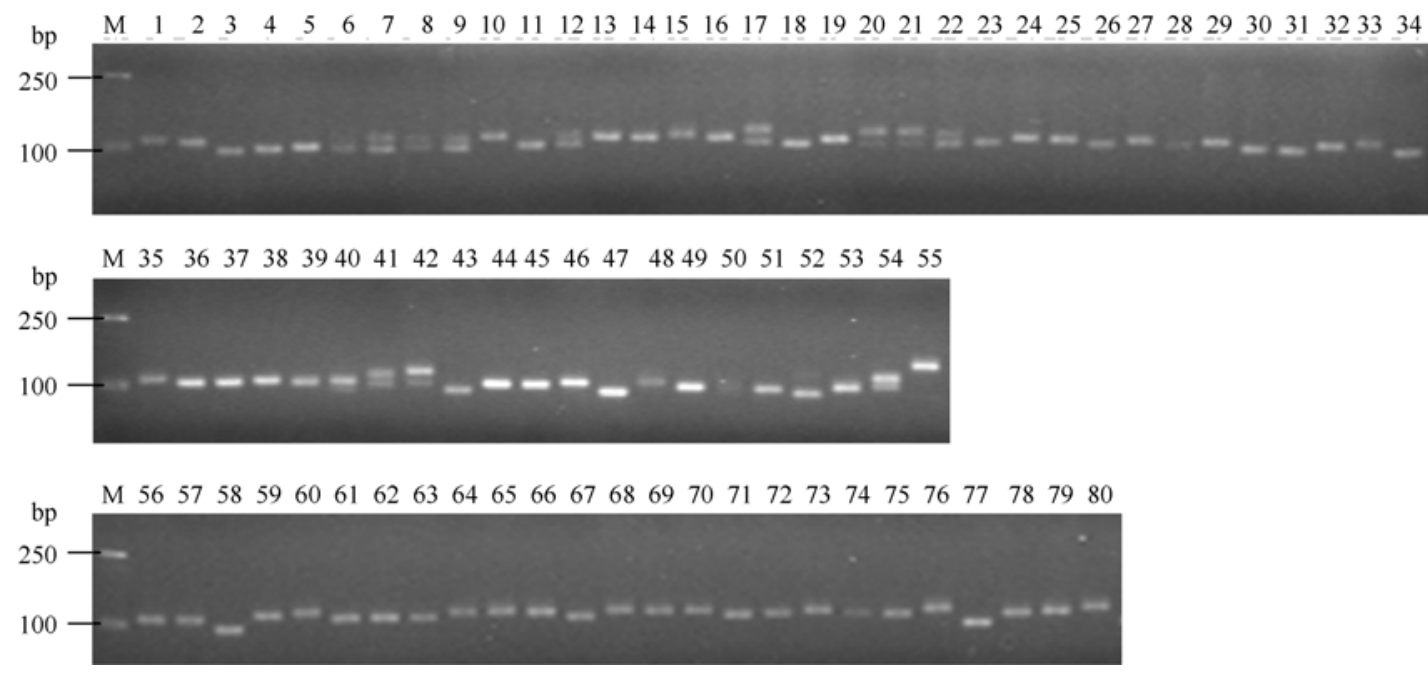

图 1 RM 286 对亚洲栽培稻和普通野生稻样品扩增电泳图谱

Fig. 1 PCR product from the cultivated rice and common wild rice accessions amplified by the primer pair RM286 图中数字为各水稻材料的编号同表 1 和表 $2, \mathrm{M}$ 表示 DNA marker DL2000。

Numbers for each lane correspond with the code for each accession given in Tables 1 and 2. M indicates DNA marker DL2000.

表 4 野生稻和栽培稻物种的遗传多样性

Table 4 Genetic diversity in common wild rice and cultivated rice

\begin{tabular}{lccccccc}
\hline $\begin{array}{c}\text { 物种或亚种名 } \\
\text { Species or subspecies }\end{array}$ & $\begin{array}{c}\text { 品系数 } \\
\text { No. of accessions }\end{array}$ & $\begin{array}{c}\text { 多态性条带数 } \\
\text { NPB }\end{array}$ & $\begin{array}{c}\text { 多态性百分比 } \\
\text { PPB (\%) }\end{array}$ & Na & Ne & $h$ & $I$ \\
\hline O. nivara & 23 & 264 & 88.59 & 1.6798 & 1.3002 & 0.1887 & 0.2960 \\
O. rufipogon & 32 & 278 & 93.28 & 1.7273 & 1.3023 & 0.1937 & 0.3070 \\
O. sativa (indica) & 14 & 182 & 61.07 & 1.4071 & 1.1688 & 0.1061 & 0.1674 \\
O. sativa (japonica) & 11 & 178 & 59.73 & 1.3953 & 1.1863 & 0.1148 & 0.1788 \\
总计 Total & 80 & 298 & 98.68 & 1.8024 & 1.2968 & 0.1905 & 0.3048 \\
\hline
\end{tabular}

NPB: number of polymorphic bands; PPB: percentage of polymorphic bands; $N a$ : observed number of alleles; $N e$ : effective number of alleles; $h$ : Nei's genetic diversity; I: Shannon's information.

\section{3 野生稻及亚洲栽培稻各样品间的遗传关系} 分析

根据 56 对 SSR 引物扩增位点构建的多态性数 据矩阵, 计算出亚洲栽培稻和野生稻各样本间的配 对遗传相似系数, 其变化范围为 $0.77 \sim 0.96$ 。再根据 所有水稻材料间的遗传相似系数利用 UPGMA 法构 建遗传关系树状图(图 2)。由图可以看出, 所有供试 材料大致分成 7 个类群, 分别来自越南和老挝的各一 个多年生普通野生稻样本(106166 和 106161)与其供 试材料关系较远而构成了树状图的基部类群(第 I 类 群), 基于两者与其供试材料间的关系, 本研究倾向 将其作为一个原始型多年生普通野生稻类群; 第 II 类群为一年生和多年生普通野生稻组成的复合群 体, 主要由来自南亚和东南亚的 6 个 $O$. nivara 和 4 个O.rufipogon样本构成, 这可能是多年生普通野生 稻在演化成一年生普通野生稻过程中的一个中间类 群; 第 III 类群主要是由 17 个 O. nivara 构成的一年
生普通野生稻类群; 第 IV 和第 V 类群分别由 5 份和 19 份多年生普通野生稻组成，鉴于其与栽培稻粳稻 和籼稻亚种较近的聚类关系, 而将它们分别作为偏 粳型和偏籼型多年生普通野生稻类群; 除粳稻品种 C418 与籼稻类群聚在一起外, 亚洲栽培稻的 indica 和 japonica 样本, 各自聚为一个组群(即第 VI 和第 VII 类群), 表明两个亚洲栽培稻亚种 (indica 和 japonica)遗传分化非常明显, 粳稻品种 C418 与籼稻 类群聚为一群是由于 $\mathrm{C} 418$ 在选育过程中并入较多 的籼稻遗传背景。此外, 从树状图上也可以看出, 野 生稻类群(包括 O. rufipogon 和 O. nivara)表现出比栽 培稻类群(包括 indica 和 japonica) 更为丰富的遗传多 样性, 其相似系数的变化范围在 $0.79 \sim 0.95$ 之间, 明 显大于栽培稻的 0.86 0.96。

\section{3 讨论}

从传统分类学角度来看, 亚洲栽培稻的 indica 


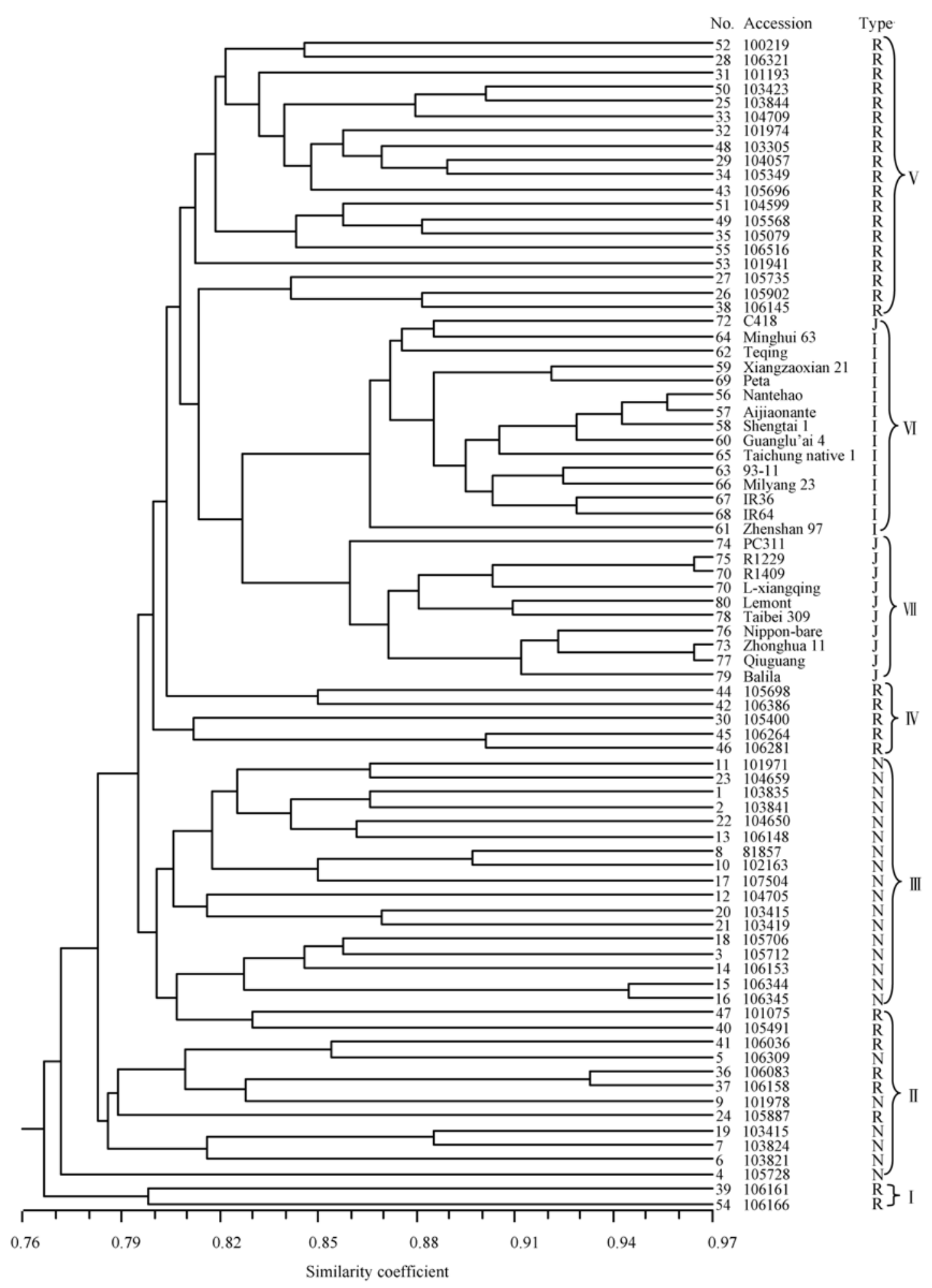

图 280 份亚洲栽培稻和野生稻样品构建的遗传关系树状图

Fig. 2 A dendrogram of 80 Asian cultivated rice and wild rice accessions illustrating genetic relationships I: indica, J: japonica, R: O. rufipogon, N: O. nivara.

和 japonica 类群无论从形态学和生活史特征上均存 在明显的差异, 将其作为两个生态亚种的分类为研 究者普遍认可。普通野生稻虽然在形态学和生活史 特征上也存在明显差异, 但人工杂交结果表明它们
之间没有生殖隔离 ${ }^{[18-19]}$, 说明两者之间有着密切的 亲缘关系, 因此在其分类上至今一直颇具争议。有 些学者认为应将其划分为两个种, 即 O. rufipogon Griff.和 O. nivara Sharma et Shastry ${ }^{[20-21]}$; 也有些学 
者认为应该将后者划归前者 ${ }^{[18]}$ 。本研究聚类结果显 示, 大部分的 O. rufipogon 和 O. nivara 样本各自聚 在一起, 说明它们的分化明显, 但在聚类结果中却 有 4 份 O. rufipogon 和 6 份 O. nivara 样本聚在一起, 是否可以认为这是一个由多年生和一年生普通野生 稻构成的复合类群, 并且可能处于原始型多年生普 通野生稻分化为偏籼偏粳多年生普通野生稻与一年 生普通野生稻的过渡阶段, 这也进一步说明这两个 类群间密切的遗传关系。但从遗传学的角度, 笔者 支持将其作为不同物种的观点 ${ }^{[21-23]}$ 。

野生稻(包括 O. rufipogon 和 O. nivara) 是亚洲栽 培稻的祖先种的观点为研究者普遍接受, 两者具有 密切的亲缘关系。近年来, 许多研究者对亚洲栽培 稻和野生稻的遗传分化及多样性关系的广泛评价大 致表明，亚洲栽培稻基因多样性下降，杂合度降低， 等位基因数明显减少 ${ }^{[3-7,10]}$ 。本研究结果显示, 普通 野生稻群体(包括 O. rufipogon 和 O. nivara) 具有比 亚洲栽培稻更丰富的遗传多样性, 这种结果还表现 在各类群的杂合子比例上，野生稻具有高达 $7.2 \%$ 的 杂合位点, 远高于栽培稻(约 $2.1 \%$ )。其原因可能是 野生稻, 特别是多年生的 O. rufipogon 具较高的异 交率。而亚洲栽培稻, 在其野生祖先种驯化过程中, 经过自然选择和人工选择而成为一种严格自花授粉 作物, 杂合度降低, 遗传多样性下降, 遗传基础变 窄。这一现象在以往的水稻遗传研究中也多次被报 道 ${ }^{[5,24-25]}$ 。因此, 加强对野生稻遗传多样性的保护, 发掘野生稻中优异的基因资源, 对水稻种质资源的 开发与利用以及水稻品种的遗传改良无疑具有非常 重要的意义。

亚洲栽培稻籼粳亚种的起源与分化问题依然存 在诸多争议。目前主要存在两种假说：(i)单源起源 假说 (monophyletic origin), 认为亚洲栽培稻的 indica 和 japonica 是野生稻经人工驯化成栽培稻以 后才开始分化的, 二者的差异在野生稻中并不存在

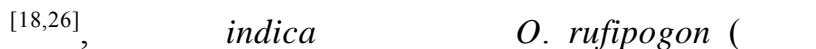
O. nivara)驯化而来, 而 japonica 则是由 indica 进一 步分化出的一种适应高海拔高纬度地区的生态型

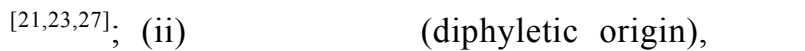
indica 和 japonica 各自独立起源于不同的普通野生 稻(包括 O. rufipogon 和 O. nivara) 群体 ${ }^{[25,28-30]}$ 。孙传 清等 ${ }^{[31]}$ 通过对普通野生稻和亚洲栽培稻的基因组 RFLP 研究表明, 除少数原始型普通野生稻外, 大多 数的国内外普通野生稻都发生了籼粳分化，但是与
栽培稻相比普通野生稻的籼粳分化是十分微小和初 步的。本研究的聚类结果显示, 亚洲栽培稻与多年 生普通野生稻(O.rufipogon)的遗传关系更为密切, 推测栽培稻的 indica 和 japonica 均可能源自多年生 普通野生稻。同时从聚类模式来看，原始型多年生 普通野生稻在进化过程中可能产生了偏籼型和偏粳 型多年生普通野生稻的分化。因此, 本研究也认为 栽培稻的 indica 和 japonica 可能分别从不同的多年 生野生稻分化而来，遵循异源起源的分化模式，即 粳型普通野生稻演化为粳稻，籼型普通野生稻演化 为籼稻 ${ }^{28]}$ 。

\section{4 结论}

野生稻比亚洲栽培稻(包括 indica 和 japonica) 具更丰富的遗传多样性; 野生稻的两个生态类群, $O$. rufipogon 和 O. nivara，尽管关系非常密切，但存在 明显的遗传分化，应将其作为两个物种来分类; 许 多普通野生稻中已发生了籼粳分化，虽然不很明显， 然而亚洲栽培稻籼粳分化是明显的；亚洲栽培稻的 indica 和 japonica 为异源起源, 均源自多年生野生 稻。

\section{References}

[1] Normile D. Archaeolgy: Yangtze seen as earliest rice site. Science, 1997, 275: 309

[2] Morishima H, Oka H I. Phylogenetic differentiation of cultivated rice. XXII. Numerable evaluation of the indica-japonica differentiation. Jpn J Breed, 1981, 31: 402-413

[3] Oka H I, Morishima H. Phylogenetic differentiation of cultivated rice: 23. Potentiality of wild progenitors to evolve the indica and japonica types of rice cultivars. Euphytica, 1982, 31: 41-50

[4] Second G. Evolutionary relationships in the sativa group of Oryza based on isozyme data. Genet Selection Evol, 1985, 17: 89-114

[5] Sun C Q, Wang X K, Li Z C, Yoshimura A, Iwata N. Comparison of the genetic diversity of common wild-rice (Oryza rufipogon Griff.) and cultivated rice (O. sativa L.) using RFLP markers. Theor Appl Genet, 2001, 102: 157-162

[6] Huang Y-H(黄燕红), Cai H-W(才宏伟), Wang X-K(王象坤). Study on different centers of origin of Asian cultivated rice. $J$ Plant Genet Res (植物遗传资源学报), 2003, 4(3): 185-190 (in Chinese with English abstract)

[7] Zhu Q H, Zheng X M, Luo J C, Gaut B S, Ge S. Multilocus analysis of nucleotide variation of Oryza sativa and its wild relatives: Severe bottleneck during domestication of rice. Mol Biol Evol, 2007, 24: 875-888

[8] McCouch S R, Chen X, Panaud O, Temnykh S, Xu Y, Cho Y G Huang N, Ishii T, Blair M. Microsatellite marker development, 
mapping and applications in rice genetics and breeding. Plant Mol Biol, 1997, 35: 89-99

[9] Tenmykh S, Park W D, Ayres N, Cartinhour S, Hanch N, Lipovich L, Cho Y G, Ishii T, McCouch S R. Mapping and genome organization of microsatellite sequence in rice (Oryza sativa L.). Theor Appl Genet, 2000, 100: 697-712

[10] Zhu Z-F(朱作峰), Sun C-Q(孙传清), Fu Y-C(付永彩), Zhang $\mathrm{P}-\mathrm{J}($ 张培江), Wang X-K(王象坤). Comparison of the genetic diversity of common wild rice and cultivated rice using SSR markers. Sci Agric Sin (中国农业科学), 2002, 35(12): 1437-1441 (in Chinese with English abstract)

[11] Ren F G, Lu B R, Li S Q, Huang J Y, Zhu Y G. A comparative study of genetic relationships among the AA-genome Oryza species using RAPD and SSR markers. Theor Appl Genet, 2003, 108: $113-120$

[12] Doyle J J, Doyle J L. A rapid DNA isolation procedure for small quantities of fresh leaf material. Phytochem Bull, 1987, 19: 11-15

[13] Nei M. Estimation of average heterozygosity and genetic distance from a small number of individuals. Genetics, 1978, 89: 583-590

[14] Yeh F C, Boyle T, Yang R C, Ye Z, Xiyan J M. POPGENE, the User Friendly Shareware for Population Genetic Analysis Version 1.31. University of Alberta and Centre for International Forestry Research, 1999

[15] Rohlf F J. NTSYS-pc, Numerical Taxonomy and Multivariate Analysis System, Version 2.02. Exeter Software, Setauket, New York, 1998

[16] Bostein D, White R L, Skolnick M, Davis R W. Construction of genetic linkage map in man using restriction fragment length polymorphism. Am J Hum Genet, 1980, 32: 314-331

[17] Anderson J A, Churchill G A, Autrique J E, Tanksley S D, Sorrells M E. Optimizing parental selection for genetic linkage maps. Genome, 1993, 36: 181-186

[18] Oka H I. Origin of Cultivated Rice. Tokyo: Japan Scientific Societies Press, 1988

[19] Lu B R, Zheng K L, Qian H R, Zhuang J Y. Genetic differentiation of wild relatives of rice as referred by the RFLP analysis. Theor Appl Genet, 2002, 106: 101-106

[20] Morishima H. Evolution and domestication of rice. In: Khush G
S, Brar D S, Hardy B, eds. Rice Genetics IV. Proceedings of the Fourth International Rice Genetics Symposium. Los Banos, the Philippines: International Rice Research Institute, 2001. pp 63-78

[21] Nayar N M. Origin and cytogenetics of rice. Adv Genet, 1973, 17: 153-292

[22] Vaughan D A. The genus Oryza L.: Current status of taxonomy. IRRI Res. Paper. Ser. 138. Los Banos, Philippines: International Rice Research Institute, 1989

[23] Chang T T. The origin, evolution, cultivation, dissemination, and diversification of Asian and African rice. Euphytica, 1976, 25: 435-441

[24] Duan S-H(段世华), Mao J-N(毛加宁), Zhu Y-G(朱英国). Genetic variation of main restorer lines of hybrid rice in China was revealed by microsatellite markers. Acta Genet Sin (遗传学报), 2002, 29(3): 250-254 (in Chinese with English abstract)

[25] Cheng C Y, Motohashi R, Ohtsubo E. Polyphyletic origin of cultivated rice: based on the interspersion pattern of SINEs. Mol Biol Evol, 2003, 20: 67-75

[26] Wang Z Y, Second G, Tanksley S D. Polymorphism and phylogenetic relationships among species in the genus Oryza as determined by analysis of nuclear RFLPs. Theor Appl Genet, 1992, 83: $565-581$

[27] Ding Y(丁䓉). The origin and evolution of cultivated rice in China. Acta Agric Sin (农业学报), 1957, 8(3): 243-260 (in Chinese)

[28] Second G. Origin of the genetic diversity of cultivated rice: study of the polymorphism scored at 40 isozyme loci. Jpn J Genet, 1982, 57: 25-57

[29] Zhu Q H, Ge S. Phylogenetic relationships among A-genome species of the genus Oryza revealed by intron sequences of four nuclear genes. New Phytol, 2005, 67: 49-265

[30] Garris A J, Tai T H, Coburn J, Kresovich A, McCouch S. Genetic structure and diversity in Oryza sativa L. Genetics, 2005, 169: 1631-1638

[31] Sun C-Q(孙传清), Wang X-K(王象坤), Yoshimura A, Iwata N. Genetic differentiation of nuclear genome in common wild rice and cultivated rice. Sci Agric Sin (中国农业科学), 1997, 30(4): 39-44 (in Chinese with English abstract) 